\title{
Exercise intensity prescription in cancer survivors: ventilatory and lactate thresholds are useful submaximal alternatives to $\mathrm{VO}_{2 \text { peak }}$
}

\author{
Justine Schneider ${ }^{1}$ (D) $\cdot$ Kathrin Schlüter ${ }^{1,2} \cdot$ Tanja Sprave $^{3} \cdot$ Joachim Wiskemann $^{1}$ (D) $\cdot$ Friederike Rosenberger $^{1,4}$ (D)
}

Received: 13 December 2019 / Accepted: 6 March 2020 / Published online: 16 March 2020

(C) The Author(s) 2020

\begin{abstract}
Purpose Most studies with cancer survivors use percentages of peak oxygen uptake $\left(\mathrm{VO}_{2 \text { peak }}\right)$ for intensity prescription. Lactate or ventilatory thresholds might be useful submaximal alternatives, but this has never been investigated. Therefore, we aimed at comparing three training sessions prescribed using $\% \mathrm{VO}_{2 \text { peak }}$ (reference), lactate thresholds, and ventilatory thresholds in terms of meeting the vigorous-intensity zone, physiological, and psychological responses.

Methods Twenty breast ( $58 \pm 10$ years) and 20 prostate cancer survivors ( $68 \pm 6$ years), $3.6 \pm 2.4$ months after primary therapy, completed a maximal cardiopulmonary exercise test and three vigorous training sessions in randomized order: 38 min of cycling at $70 \% \mathrm{VO}_{2 \text { peak }}\left(\mathrm{M}-\mathrm{VO}_{2 \text { peak }}\right), 97 \%$ of individual anaerobic lactate threshold (M-IAT), and $67 \%$ between ventilatory thresholds 1 and 2 (M-VT). Heart rate (HR), blood lactate concentration (bLa), perceived exertion, and enjoyment were assessed.

Results Cancer survivors exercised at $75 \pm 23,85 \pm 18$, and $79 \pm 19 \mathrm{~W}$ during $\mathrm{M}-\mathrm{VO}_{2 \text { peak }}, \mathrm{M}-\mathrm{IAT}$, and M-VT $(p>.05)$. Sessions could not be completed in 3,8 , and 6 cases. Session completers showed HR of $82 \pm 7,83 \pm 9$, and $84 \pm 8 \% \mathrm{HR}_{\text {peak }}$ and bLa of 3.7 $\pm 1.9,3.9 \pm 0.9$, and $3.9 \pm 1.5 \mathrm{mmol} \cdot \mathrm{l}^{-1}$, which was not different between sessions $(p>.05)$. However, variance in bLa was lower in M-IAT compared to $\mathrm{M}-\mathrm{VO}_{2 \text { peak }}(p=.001)$ and to M-VT $(p=.022)$.

Conclusion All intensity prescription methods on average met the targeted intensity zone. Metabolic response was most homogeneous when using lactate thresholds.

Implications for cancer survivors Submaximal thresholds are at least as useful as $\mathrm{VO}_{2 \text { peak }}$ for intensity prescription in cancer survivors. Overall, slightly lower percentages should be chosen to improve durability of the training sessions.
\end{abstract}

Keywords Aerobic training $\cdot$ Endurance training $\cdot$ Oncology $\cdot$ Peak oxygen uptake $\cdot$ Lactate threshold $\cdot$ Ventilatory threshold

Friederike Rosenberger

friederike.rosenberger@nct-heidelberg.de

$1 \quad$ Working Group Exercise Oncology, Department of Medical Oncology, National Center for Tumor Diseases (NCT), Heidelberg University Hospital, Im Neuenheimer Feld 460, 69120 Heidelberg, Germany

2 Institute of Sports and Sport Science, Heidelberg University, Heidelberg, Germany

3 Department of Radiation Oncology, University Hospital Heidelberg and National Center for Radiation Oncology (NCRO), Heidelberg Institute for Radiation Oncology (HIRO), Heidelberg, Germany

4 Division of Health Sciences, German University of Applied Sciences for Prevention and Health Management (DHfPG),

Saarbrücken, Germany

\section{Introduction}

Exercise is recommended for cancer survivors by expert panels worldwide because of its various beneficial effects [1-4]. Today, it represents a recognized part of supportive therapy. Regarding aerobic activity, $150 \mathrm{~min}$ of moderate or $75 \mathrm{~min}$ of vigorous-intensity exercise or an equivalent combination per week is recommended [1,2]. Meeting these zones of moderate or vigorous intensity is crucial to elicit the intended training effects and avoid underload or overload of patients.

Some expert panel recommendations for cancer survivors do not specify how to target different intensity zones [1]. Others suggest $60-85 \%$ of oxygen uptake reserve $\left(\mathrm{VO}_{2} \mathrm{R}\right)$ or $60-85 \%$ of heart rate reserve (HRR) or $>70 \%$ of peak heart rate $\left(\mathrm{HR}_{\text {peak }}\right)$ to target the vigorous-intensity zone $[5,6]$. However, percentages of $\mathrm{VO}_{2} \mathrm{R}$ are rarely used due to the effort of resting oxygen uptake measurements. Furthermore, an analysis of cardiopulmonary exercise tests (CPETs) 
showed that percentages of HRR result in an intensity overload in cancer survivors due to their elevated resting heart rate $[6,7]$, whereas percentages of $\mathrm{HR}_{\text {peak }}$ appear appropriate [6, 8]. Most studies in cancer survivors prescribe certain percentages of peak oxygen uptake $\left(\mathrm{VO}_{2 \text { peak }}\right)$ to target intensity zones $[9,10]$. However, all relative percent concepts can be criticized for assuming that a fixed percentage of a reference value represents the same intensity in all individuals. These percentages might fit on average, but be imprecise on an individual level.

Individual threshold concepts, i.e., blood lactate (bLa) or ventilatory thresholds $[11,12]$, which are anchored to the individual metabolic profile, are considered more accurate than fixed percentages of reference values [13, 14]. Furthermore, their determination does not require maximal exhaustion which appears valuable in cancer survivors who are not able or willing to spend maximal effort. Threshold concepts have a long tradition in high-performance and recreational sports due to their capability of maximizing physical performance [14-16] and are also considered superior in maximizing beneficial effects of exercise in cancer survivors [6]. However, research on threshold concepts for intensity prescription in cancer survivors is scarce. So far, ventilatory thresholds have only been used for prescribing low to moderate but no vigorous-intensity exercise [17-19], whereas bLa thresholds have not been used for intensity prescription in cancer survivors yet. Their determination usually requires completing at least five stages of a stepwise incremental exercise protocol [20] which is not possible for many cancer survivors due to their low aerobic fitness $[6,21]$. However, it appears worth testing whether bLa thresholds can be determined in a CPET protocol with 1-min stages $[6,21]$ and used for intensity prescription in cancer survivors.

Therefore, the present study aimed at comparing three vigorous training sessions in terms of durability, physiological, and psychological responses: (a) a session prescribed in percentages of $\mathrm{VO}_{2 \text { peak }}$ which, as the most commonly used method, served as reference here; (b) a session prescribed by means of ventilatory thresholds; and (c) for the first time with cancer survivors, a session prescribed by means of bLa thresholds, all determined from one CPET. It was hypothesized that the threshold-based methods meet the vigorous-intensity zone as successfully as percentages of $\mathrm{VO}_{2 \text { peak }}$ but elicit a more homogeneous metabolic response as they are anchored to the individual metabolic profile. Knowledge on this will improve exercise intensity prescription for cancer survivors.

\section{Methods}

Participants A total of 40 cancer survivors, 20 with breast and 20 with prostate cancer to represent the most common types of cancer in females and males, were recruited for participation.
Participants were recruited by means of advertising flyers at the breast and prostate cancer presentations of our comprehensive cancer center and medical practices, hospitals, and selfhelp groups around. All participants met the following inclusion criteria: diagnosed with breast or prostate cancer, 6 to 52 weeks after end of primary therapy (i.e., surgery and/or radiotherapy and/or chemotherapy), 18 to 75 years of age, and no regular vigorous endurance or resistance training $(>1$ session per week) within the last 6 months. Exclusion criteria were diagnosis with additional other cancer or severe comorbidities that preclude participation in exercise testing or training (acute infectious diseases, severe cardiac, respiratory, renal, or neurological diseases).

General design Following a cross-sectional design, each patient performed four tests: a CPET and then three vigorousintensity training sessions targeted by means of the three different prescription methods in randomized order (block randomization procedure). All tests took place once per week, separated by at least 4 days to avoid training adaptations, and were conducted on electromagnetically braked cycle ergometers (Ergoselect 100 or 200, Ergoline, Bitz, Germany).

Cardiopulmonary exercise tests CPETs were preceded by a 2min resting period on the cycle ergometer. They started at $20 \mathrm{~W}$ and increased every minute by $10 \mathrm{~W}$ until volitional exhaustion. Patients were encouraged to exert maximal effort. After a 10-min rest following the CPET, each patient performed a supramaximal verification test. The protocol started at $20 \mathrm{~W}$ and work rate was rapidly manually increased to $110 \%$ peak power output (PPO) of the preceding CPET [22]. Patients were again encouraged to exert maximal effort and the test was continued until volitional exhaustion.

A 12-lead electrocardiogram was continuously monitored (CardioPart 12 Blue, Amedtec, Aue, Germany). Gas exchange data were continuously measured using a breath-by-breath gas analysis system (Ergostik, Geratherm Respiratory, Bad Kissingen, Germany). The system was calibrated prior to each test according to the manufacturer's guidelines. For bLa determination, capillary blood samples from the hyperemized (Finalgon ${ }^{\circledR}$ ) earlobe were taken at rest, at the end of each 1min increment, and after exercise cessation. They were analyzed using an enzymatic-amperometric method (Super GL compact, Hitado, Möhnesee, Germany). Blood pressure (Bp) and ratings of perceived exertion (RPE, 6 to 20 BORG scale [23]) were assessed every $2 \mathrm{~min}$.

PPO was interpolated when appropriate. $\mathrm{VO}_{2 \text { peak }}$ and $\mathrm{HR}_{\text {peak }}$ were defined as the highest 20 -s average value reached during or immediately after the CPET. Ventilatory threshold 1 (VT1) and 2 (VT2) were determined using the V-slope method $\left(\mathrm{VCO}_{2} / \mathrm{VO}_{2}\right)$ [11] as primary and the $\mathrm{VE} / \mathrm{VCO}_{2}$ method [16] as secondary criterion. The individual anaerobic bLa threshold (IAT) was determined at $1 \mathrm{mmol} \mathrm{L}^{-1}$ above minimum lactate 
equivalent (Ergonizer, Freiburg, Germany) [24]. This concept was originally designed for a 3-min exercise stage protocol. However, it was adapted here to the CPET protocol because cancer survivors' fitness levels are usually not sufficient to receive an evaluable blood lactate curve when using 3-min exercise stage protocols.

CPETs were considered maximal when $\mathrm{VO}_{2 \text { peak }}$ in the verification test did not exceed $\mathrm{VO}_{2 \text { peak }}$ in the CPET by more than $3 \%$ (verification criterion). This verification criterion represents the measurement accuracy of $\mathrm{VO}_{2}$ determination reported by the manufacturer (Ergostik, Geratherm Respiratory, Bad Kissingen, Germany). Furthermore, following secondary criteria, CPETs were considered maximal when two or more of the following criteria occurred: maximal respiratory exchange ratio $\left(\mathrm{RER}_{\text {peak }}\right) \geq 1.1$, HRpeak $\geq 200$ minus age, peak $\mathrm{bLa}\left(\mathrm{bLa}_{\text {peak }}\right) \geq 8 \mathrm{mmol} \mathrm{L}^{-1}, \mathrm{RPE} \geq 18[5,25,26]$. However, $\mathrm{VO}_{2 \text { peak }}$ from the CPET (irrespective of maximal or not) was used to derive training intensity because this is the usual procedure in literature that should serve as a reference here.

Training sessions Training sessions lasted $38 \mathrm{~min}$ to theoretically reach $75 \mathrm{~min}$ of vigorous-intensity exercise as recommended when performing two sessions per week [1]. All sessions were designed to target the vigorous-intensity zone: $70 \% \mathrm{VO}_{2 \text { peak }}[8,27]$ (method $\mathrm{VO}_{2 \text { peak }}, \mathrm{M}-\mathrm{VO}_{2 \text { peak }}$ ), slightly below (97\%) IAT $[15,16,20]$ (M-IAT), and two-thirds $(67 \%)$ between VT1 and VT2 $[11,16]$ (M-VT). Power output (W) corresponding to these points was prescribed. To assess the evoked strain, HR (Polar A300 monitor, Polar Electro Oy, Kempele, Finland), bLa, Bp, and RPE were recorded at rest and after 10, 20, 30, and 38 min of exercise. Exercise values were averaged over the four measurement time points. A lactate steady state (LASS) was defined as an increase in bLa of $\leq 0.9 \mathrm{mmol} \mathrm{L}^{-1}$ during the last $18 \mathrm{~min}$ of each training session $\left(\leq 0.05 \mathrm{mmol} \mathrm{L}^{-1} \mathrm{~min}^{-1}\right)[15,28]$. Enjoyment was assessed after each training session using a single-item 7-point Likert scale ("How much did you enjoy the training session?" $1=$ not at all to $7=$ very much) adjusted from Rogers et al. [29]. For safety assessment, adverse events were recorded.

Statistical analyses The sample size was based on a preceding similar study with healthy male participants [30]. Normality was tested using the Shapiro-Wilk test. Differences between the three training sessions for continuous data were assessed by one-way repeated measures analysis of variance (ANOVA) or in the case of non-parametric or ordinal scaled data by Friedman's ANOVA. Differences between cancer entity were calculated using independent $t$ tests or the Mann-Whitney $U$ test in the case of non-parametric or ordinal scaled data. Dependent dichotomous data were assessed using Cochran's $Q$ test with McNemar post hoc test. For independent dichotomous data, the $\chi^{2}$ test was used. The Pitman-Morgan test was used to test for differences of homogeneity of bLa and
$\% \mathrm{HR}_{\text {peak }}$ response between training sessions. Correction for multiple testing was applied using the Bonferroni-Holm post hoc test. $p<.05$ was considered significant. Data are presented as means \pm standard deviations or individual courses. All Data were analyzed using IBM SPSS Version 25 (IBM Corp, Armonk, NY) and MATLAB Version R2018a (MathWorks, Natick, MA).

\section{Results}

Participants' characteristics are shown in Table $1 . \mathrm{VO}_{2 \text { peak }}$ could not be determined in one case due to fear of wearing a facemask, IAT could not be determined in one case because of a near linear bLa curve, and thus, $\mathrm{M}-\mathrm{VO}_{2 \text { peak }}$ and M-IAT could not be performed in one case each. Seven out of the 39 CPETs with $\mathrm{VO}_{2 \text { peak }}$ measurement (18\%) were not considered maximal based on secondary criteria for maximal exhaustion. Interestingly, according to the verification test, these seven CPETs were all considered maximal, whereas 13 other CPETs did not satisfy the verification criterion (i.e., $\mathrm{VO}_{2 \text { peak }}$ reached during the verification test was more than $3 \%$ higher than $\mathrm{VO}_{2 \text { peak }}$ reached during CPET). In other words, according to the verification test, $33 \%$ of the performed CPETs were not considered maximal. Still, all patients were included in the data analyses, in order to reflect what is usually done in practice. One patient's HR had to be excluded from data analyses as a result of measurement problems.

The results of the training sessions are presented in Table 2. Prescribed absolute power output did not differ between the training sessions (all $p>.05$ ), but prescribed relative power output was lower for $\mathrm{M}-\mathrm{VO}_{2 \text { peak }}$ when compared to M-IAT $(p=.028)$ and to M-VT $(p=.036) . \mathrm{M}-\mathrm{VO}_{2 \text { peak }}, \mathrm{M}-\mathrm{IAT}$, and M-VT were terminated prematurely in 3, 8 , and 6 cases, respectively, which was not different between training sessions $(p=.093)$. Sixteen of the 17 premature terminations were due to muscular exhaustion, whereas one resulted from knee pain, which was considered as a minor adverse event. No severe adverse event occurred. When comparing \%PPO between session completers and those who terminated prematurely, there was no significant difference for $\mathrm{M}-\mathrm{VO}_{2 \text { peak }}(57$ vs. $61 \% \mathrm{PPO}$, $p=.484)$, whereas \% $\mathrm{PPO}$ of completers was significantly lower for M-IAT (62 vs. $72 \% \mathrm{PPO}, p=.006$ ) and M-VT (58 vs. $70 \% \mathrm{PPO}, p=.001)$.

For those who completed all exercise sessions, $\% \mathrm{HR}_{\text {peak }}$, homogeneity of $\% \mathrm{HR}_{\text {peak }}$, mean bLa and the number of participants who reached LASS did not differ between the training sessions (all $p>.05$ ). However, the variance of bLa during M-IAT was significantly lower compared with $\mathrm{M}-\mathrm{VO}_{2 \text { peak }}$ $(p=.001, n=30)$ and M-VT $(p=.022, n=29$, Fig. 1). RPE and enjoyment were not different between the training sessions. 
Table 1 Participants' characteristics. Data presented as mean \pm SD unless stated otherwise

\begin{tabular}{|c|c|c|c|}
\hline$n$ & $\begin{array}{l}\text { Total } \\
40\end{array}$ & $\begin{array}{l}\mathrm{BCa} \\
20\end{array}$ & $\begin{array}{l}\mathrm{PCa} \\
20\end{array}$ \\
\hline Age & $62.9 \pm 9.2$ & $58.4 \pm 9.7$ & $67.5 \pm 6.0$ \\
\hline BMI $\left(\mathrm{kg} / \mathrm{m}^{2}\right)$ & $27.4 \pm 3.9$ & $27.1 \pm 4.8$ & $27.7 \pm 2.7$ \\
\hline Time since diagnosis (months) & $20.8 \pm 29.1$ & $9.7 \pm 3.5$ & $32.0 \pm 38.2$ \\
\hline Time since end of primary treatment ${ }^{\dagger}$ (months) & $3.6 \pm 2.4$ & $3.5 \pm 2.0$ & $3.8 \pm 2.7$ \\
\hline \multicolumn{4}{|l|}{ Type of treatment received, $n(\%)$} \\
\hline Surgery & $36(90)$ & $20(100)$ & $16(80)$ \\
\hline Chemotherapy & $10(25)$ & $10(50)$ & $0(0)$ \\
\hline Radiation & $32(80)$ & $18(90)$ & $14(70)$ \\
\hline Antihormonal therapy ${ }^{*}$ & $23(58)$ & $17(85)$ & $6(30)$ \\
\hline Current $ß$-blocker intake, $n(\%)$ & $11(28)$ & $5(13)$ & $6(15)$ \\
\hline $\mathrm{VO}_{2 \text { peak }}(\mathrm{mL} / \mathrm{min} / \mathrm{kg}), n$ & $19.7 \pm 4.1,39$ & $19.2 \pm 3.4,20$ & $20.3 \pm 4.7,19$ \\
\hline PPO (W/kg) & $1.6 \pm 0.4$ & $1.5 \pm 0.3$ & $1.7 \pm 0.4$ \\
\hline
\end{tabular}

$B M I$, body mass index; $B C a$, breast cancer patients; $P P O$, peak power output; $P C a$, prostate cancer patients; $S D$, standard deviation; $V O_{2 \text { peak }}$, peak oxygen consumption

${ }^{\dagger}$ Surgery and/or radiotherapy and/or chemotherapy

$\$ 21$ of 23 participants were still undergoing antihormonal therapy at the beginning of the study

\section{Discussion}

In the present study, intensity prescription by means of bLa and ventilatory thresholds was for the first time systematically investigated in breast and prostate cancer survivors after primary therapy and compared with the widely used percentages of $\mathrm{VO}_{2 \text { peak. }}$. Our data indicate that within the examined patient population, $70 \% \mathrm{VO}_{2 \text { peak }}$, slightly below (97\%) IAT, and twothirds (67\%) between VT1 and VT2 were equally suitable to meet the vigorous-intensity zone. As expected, the variance of bLa response was smaller when bLa thresholds were used for intensity prescription, but surprisingly not when ventilatory thresholds were used. In all three training sessions, premature exercise terminations occurred, indicating that intensity was chosen slightly too high.

While prescribed absolute power output was not different between the training sessions, prescribed power output relative to the individual peak power output was higher for M-IAT and $\mathrm{M}-\mathrm{VT}$ compared with $\mathrm{M}-\mathrm{VO}_{2 \text { peak }}$. It must be noted that the used percentage within each intensity prescription method was chosen based on best knowledge and experience to meet the vigorous-intensity zone. This in a sense arbitrary choice naturally affected power output and the resulting physiological and psychological responses. This imbalance should be kept in mind when interpreting the findings. However, they could be leveled out in future by slightly adapting the used percentage within each intensity prescription method: When the early session terminations are additionally considered, $65 \% \mathrm{VO}_{2 \text { peak }}, 90 \%$ of IAT, and $60 \%$ between VT1 and VT2 (instead of $70 \%, 97 \%$, and $67 \%$ ) might be prescriptions for more durable and comparable vigorous-intensity training sessions.
For those participants who completed all training sessions, mean percentage of $\mathrm{HR}_{\text {peak }}$ corresponded to the vigorousintensity zone of $77-95 \% \mathrm{HR}_{\text {peak }}$ given by the ACSM for apparently healthy adults [27]. This prescription was shown to be also valid in breast cancer survivors at the end of primary therapy [8]. Mean bLa responses to all three training sessions were nearly $4 \mathrm{mmol} \mathrm{L}^{-1}$ which is roughly estimated to correspond to maximal LASS in untrained individuals and thus indicates that the upper limit of the vigorous training zone was met $[15,30]$. Altogether, the cardio-metabolic exercise responses indicate that the vigorous-intensity zone was met on average in all three exercise sessions.

Surprisingly, although the cardio-metabolic responses reflected vigorous intensities, RPE reflected moderate intensities according to the ACSM guidelines for apparently healthy adults (RPE 12-13) [27]. Enjoyment was also rated relatively high. However, it has to be considered that all training sessions were supervised in a one-on-one manner and albeit the supervising personal avoided conversations, some participants reported having enjoyed the undivided attention. Furthermore, socially desirable responding could have been an influencing factor. Therefore, subjective exercise responses should be interpreted cautiously.

Considering the homogeneity of physiological strain, the variance of bLa response was lower when bLa thresholds were used for intensity prescription compared with when $\mathrm{VO}_{2 \text { peak }}$ was used. This was in accordance with our hypothesis. Variability in the degree of effort in the CPET might have contributed to the heterogeneity of metabolic strain when intensity was prescribed in percentages of $\mathrm{VO}_{2 \text { peak }}$. However, heterogeneous metabolic responses at given percentages of $\mathrm{VO}_{2 \text { peak }}$ were also found in a previous study with healthy male 
Table 2 Comparison of the three exercise sessions. Intensity during sessions was prescribed as follows: $70 \% \mathrm{VO}_{2 \text { peak }}(\mathrm{M}$ $\left.\mathrm{VO}_{2 \text { peak }}\right), 97 \%$ IAT (M-IAT), and $67 \%$ between VT1 and VT2 (MVT). Data presented as mean \pm SD unless stated otherwise

\begin{tabular}{|c|c|c|c|c|}
\hline & & $\begin{array}{l}\mathrm{M}- \\
\mathrm{VO}_{2 \text { peak }}\end{array}$ & M-IAT & $\mathrm{M}-\mathrm{VT}$ \\
\hline \multirow[t]{3}{*}{ Prescribed power output (W) } & Total $(n=38)$ & $75 \pm 23$ & $85 \pm 18$ & $79 \pm 19$ \\
\hline & $\mathrm{BCa}(n=19)$ & $64 \pm 17 * \dagger$ & $71 \pm 9 * \dagger$ & $70 \pm 16^{* \dagger}$ \\
\hline & $\mathrm{PCa}(n=19)$ & $86 \pm 24 * \dagger$ & $98 \pm 16^{* \dagger}$ & $88 \pm 19 *{ }^{\dagger}$ \\
\hline \multirow[t]{3}{*}{ Prescribed relative power output (\%PPO) } & total $(n=38)$ & $57 \pm 9$ & $64 \pm 9 *$ & $61 \pm 8^{*}$ \\
\hline & $\mathrm{BCa}(n=19)$ & $57 \pm 10$ & $62 \pm 7$ & $62 \pm 9$ \\
\hline & $\mathrm{PCa}(n=19)$ & $57 \pm 9$ & $66 \pm 11$ & $59 \pm 8$ \\
\hline \multirow[t]{3}{*}{ Number of premature session terminations } & Total $(n=38)$ & 3 & 8 & 6 \\
\hline & $\mathrm{BCa}(n=19)$ & 2 & 3 & 4 \\
\hline & $\mathrm{PCa}(n=19)$ & 1 & 5 & 2 \\
\hline \multirow[t]{3}{*}{ Relative heart rate $\left(\% \mathrm{HR}_{\text {peak }}\right)$} & Total $(n=28)^{\ddagger}$ & $82 \pm 7$ & $83 \pm 9$ & $84 \pm 8$ \\
\hline & $\mathrm{BCa}(n=14)$ & $84 \pm 6$ & $84 \pm 6$ & $87 \pm 6$ \\
\hline & $\mathrm{PCa}(n=14)$ & $80 \pm 7$ & $83 \pm 12$ & $81 \pm 8$ \\
\hline \multirow[t]{3}{*}{ Blood lactate concentration $\left(\mathrm{mmol} \mathrm{L}^{-1}\right)$} & Total $(n=28)^{\ddagger}$ & $3.7 \pm 1.9$ & $3.9 \pm 0.9$ & $3.9 \pm 1.5$ \\
\hline & $\mathrm{BCa}(n=14)$ & $4.1 \pm 1.8$ & $4.2 \pm 1.1$ & $4.5 \pm 1.1^{\dagger \dagger}$ \\
\hline & $\mathrm{PCa}(n=14)$ & $3.4 \pm 2.0$ & $3.6 \pm 0.7$ & $3.3 \pm 1.6^{* \dagger}$ \\
\hline \multirow{3}{*}{$\begin{array}{l}\text { Proportion of participants } \\
\text { attaining LASS }{ }^{\S}(\%)\end{array}$} & Total $(n=36,31,34)$ & 92 & 97 & 91 \\
\hline & $\mathrm{BCa}(n=18,16,16)$ & 90 & 80 & 94 \\
\hline & $\mathrm{PCa}(n=18,15,18)$ & 83 & 93 & 89 \\
\hline \multirow[t]{3}{*}{ RPE breathing (scale 6-20) } & Total $(n=28)^{\ddagger}$ & $12.7 \pm 2.3$ & $12.9 \pm 1.8$ & $12.8 \pm 2.2$ \\
\hline & $\mathrm{BCa}(n=14)$ & $13.4 \pm 2.0$ & $13.4 \pm 2.0$ & $13.6 \pm 2.6^{* \dagger}$ \\
\hline & $\mathrm{PCa}(n=14)$ & $11.9 \pm 2.4$ & $12.5 \pm 1.5$ & $12 \pm 1.4^{* \dagger}$ \\
\hline \multirow[t]{3}{*}{ RPE legs (scale 6-20) } & Total $(n=28)^{*}$ & $12.8 \pm 2.2$ & $13.3 \pm 2.1$ & $13.0 \pm 2.4$ \\
\hline & $\mathrm{BCa}(n=14)$ & $13.4 \pm 2.0$ & $13.9 \pm 2.4$ & $13.9 \pm 2.6^{* \dagger}$ \\
\hline & $\mathrm{PCa}(n=14)$ & $12.3 \pm 2.4$ & $12.7 \pm 1.5$ & $12.1 \pm 1.9 * \dagger$ \\
\hline \multirow[t]{3}{*}{ Enjoyment (scale 1-7) } & Total $(n=28)^{*}$ & $5.5 \pm 1.4$ & $5.5 \pm 1.4$ & $5.3 \pm 1.3$ \\
\hline & $\mathrm{BCa}(n=14)$ & $5.1 \pm 1.7$ & $5.3 \pm 1.8$ & $5.1 \pm 1.5$ \\
\hline & $\mathrm{PCa}(n=14)$ & $5.9 \pm 0.9$ & $5.8 \pm 0.9$ & $5.6 \pm 0.9$ \\
\hline
\end{tabular}

$B C a$, breast cancer patients; $P C a$, prostate cancer patients; $L A S S$, lactate steady state; $R P E$, rating of perceived exertion; $S D$, standard deviation

*Significant difference compared with $\mathrm{M}-\mathrm{VO}_{2 \text { peak }}: \mathrm{p}<0.05$

${ }^{*}$ Significant difference between cancer entities: $p<0.05$

${ }^{*}$ Reduction from $n=38$ to $n=28$ due to exclusion of data from participants with early session terminations

$\S$ Proportion of participants who attained LASS among those who completed the respective exercise session participants who reached maximal exhaustion during CPET [30]. The authors therefore advised against using $\% \mathrm{VO}_{2 \text { peak }}$ for training prescription, although in that study, $\% \mathrm{VO}_{2 \text { peak }}$ was not compared with other intensity prescription methods. Surprisingly, variance in bLa response was not as low when ventilatory thresholds were used. This might be attributable to the more challenging determination of ventilatory thresholds as reflected by a lower inter- and intra-evaluator agreement compared with lactate thresholds [31].

In terms of cardiocirculatory strain, there were no statistically significant differences in the variance of heart rate response. However, single participants (outliers) demonstrated heart rates above $100 \% \mathrm{HR}_{\text {peak }}$ only in the sessions prescribed by means of bLa and ventilatory thresholds and not in the session prescribed by means of $\mathrm{VO}_{2 \text { peak }}$ (Fig. 1). Based on this, one might conclude that percentages of $\mathrm{VO}_{2 \text { peak }}$ (or maximal values in general) for intensity prescription are superior to elicit a homogeneous cardiocirculatory strain while bLa thresholds for intensity prescription are superior to elicit a homogeneous metabolic strain. While this appears obvious, confirmatory studies are to the best of our knowledge missing.

Although bLa thresholds have never been used before for intensity prescription in cancer survivors, they represent a general method for individually tailored exercise prescription in high-performance sports [15]. The challenge in the present study was to determine bLa thresholds from a CPET appropriate for cancer survivors. Typically, stepwise incremental exercise protocols with 3-min stages are used and at least five stages are needed for bLa threshold determination $[12,15$, 20]. These five stages were easily reached in the present 

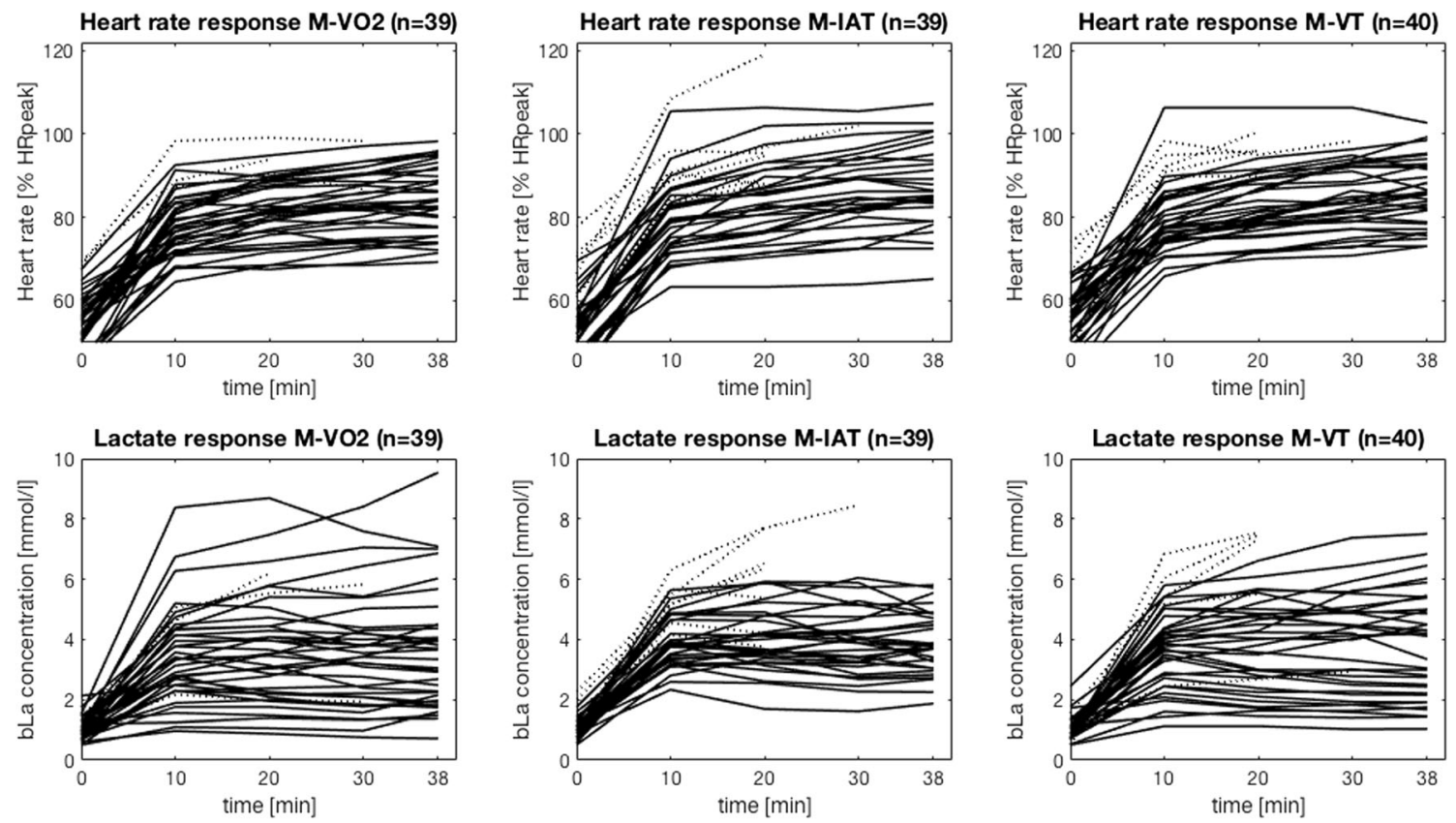

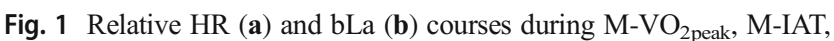
and M-VT (from left to right). Intensity during sessions was prescribed as follows: $70 \% \mathrm{VO}_{2 \text { peak }}\left(\mathrm{M}-\mathrm{VO}_{2 \text { peak }}\right), 97 \%$ IAT (M-IAT), and $67 \%$ between VT1 and VT2 (M-VT). Dotted lines show courses of

participants who terminated the session prematurely. The $\% \mathrm{HR}_{\text {peak }}$ course of one patient is not displayed for $\mathrm{M}-\mathrm{VO}_{2 \text { peak }}$ due to a measurement problem

CPET protocol with 1-min stages and the resulting IAT proved useful for intensity prescription - if needed with the above mentioned intensity reduction to $90 \%$ IAT. Lactate thresholds constitute the advantage that maximal exhaustion during CPET is not required. This is deemed particularly useful since in the present study $10 \%$ and $33 \%$ of the conducted CPETs were not considered maximal based on secondary criteria for maximal exhaustion and on the verification test, respectively. Altogether, the IAT derived from a CPET appears useful in cancer survivors which should be further investigated through training intervention studies.

To our knowledge, ventilatory thresholds have not yet been used for prescribing vigorous-intensity exercise in cancer survivors and only three prior studies with cancer survivors did so for prescribing low- to moderate-intensity exercise [17-19]. These studies showed that cardiorespiratory fitness improved after 27 weeks [17], but not after 18 weeks [18, 19] of training at the VT1 performed thrice or twice per week, respectively. Since the intervention groups were compared with non-exercising control groups, no conclusion can be drawn on whether the missing effects were owing to the low exercise stimulus at the VT1 or to the method of intensity prescription itself. Results from studies with healthy participants suggest that moderate to vigorous exercise prescribed by means of ventilatory thresholds elicit superior training adaptations compared with a relative percent concept $[14,32]$. More precisely, $100 \%$ of the participants who performed 12 weeks of training prescribed by means of ventilatory thresholds demonstrated an improvement in $\mathrm{VO}_{2 \text { peak }}$, whereas

only $42 \%$ [14] and $60 \%$ [32] of those following the same intervention based on percentages of HRR were able to improve their $\mathrm{VO}_{2 \text { peak }}$. Altogether, ventilatory thresholds appear suitable for prescribing also vigorous-intensity exercise in cancer survivors.

\section{Limitations}

The strongest limitation of the present study is the somewhat arbitrary choice of percentages within the prescription methods. This cannot be avoided and although it limits direct comparability of the three training sessions, it does not hamper the conclusions drawn from this study. Furthermore, these findings (including the suggested adaptions of percentages within each intensity prescription method) are prerequisite for implementing the intensity prescription methods into training intervention studies. Another limitation is that threshold concepts might be considered somewhat sophisticated with regard to clinical practice. Yet, as a first approach, we sought to systematically evaluate these methods that are appreciated in elite sports and can be determined without attaining maximal exhaustion in a CPET. In a second approach, it would be interesting to compare these highly objective methods to others that are easier to use, including subjective methods based, e.g., on RPE.

Furthermore, the fact that we did not exclude all patients who had not attained their "true $\mathrm{VO}_{2 \max }$ " during CPET might be a limitation, since spending maximal effort during CPET is required for an adequate application of $\% \mathrm{VO}_{2 \text { peak }}$ for intensity 
prescription. However, the validity of secondary criteria for maximal exhaustion has been strongly criticized already for healthy individuals $[33,34]$ and their applicability has never been assessed in cancer survivors. And, as already mentioned above, we decided to include all patients in the data analyses as this more realistically reflects practice in previous research. Since there was no concordance between the two methods (secondary criteria for maximal exhaustion and verification test) regarding the number of patients who failed to attain maximal exhaustion, it is questionable whether secondary criteria are even useful for cancer survivors. Furthermore, $33 \%$ not attaining "real $\mathrm{VO}_{2 \max }$ " during CPET raises the question of whether $\% \mathrm{VO}_{2 \text { peak }}$ is an appropriate intensity prescription method in cancer survivors, since in the present study, one-third would have exercised at a too low intensity with this method. Even though this assumption is not reflected by the results, it could have more pronounced consequences regarding training responses for interventions with longer durations and/or in larger cohorts.

Finally, it should be noted that sample size was derived from a similar study and not calculated based on expected effects because there were no reference points available. The study has therefore pilot character and further confirmatory research is needed. In addition, the findings are not necessarily transferable to patients with entities other than breast and prostate cancer or to those undergoing anti-cancer treatment.

\section{Conclusion}

In the present study, three training sessions prescribed by means of percentages of $\mathrm{VO}_{2 \text { peak }}$ (reference), blood lactate thresholds, and ventilatory thresholds were compared in terms of durability, physiological, and psychological responses in breast and prostate cancer survivors after primary treatment. There were no significant differences in the number of premature terminations. The vigorous-intensity zone was met on average through all three intensity prescription methods as indicated by cardio-metabolic responses. Blood lactate thresholds appear most suitable for training prescription if a defined metabolic strain is intended because this method elicited the most homogeneous blood lactate response. All three exercise sessions were equally enjoyed and rated as moderate despite their vigorous intensity. Altogether, submaximal thresholds are at least as useful as $\mathrm{VO}_{2 \text { peak }}$ for intensity prescription in breast and prostate cancer survivors after primary treatment. To avoid early session terminations, slightly lower percentages of the reference points might be preferable, e.g., $65 \%$ $\mathrm{VO}_{2 \text { peak }}, 90 \%$ IAT, and $60 \%$ between VT1 and VT2.

Acknowledgments We thank the Dietmar Hopp Foundation for funding the study. Furthermore, we thank the German Cancer Research Center (DKFZ) for the study assessment support. Prof. Dr. Kai Röcker
(Furtwangen University, Germany) is thanked for the fruitful discussion about CPET protocols and lactate threshold determination in cancer survivors. And finally, we thank all participants for their dedication to the study.

Author's contribution F.R. and J.W. designed the study. F.R., J.W., K.S., J.S., and T.S. contributed to data acquisition. J.S. and F.R. analyzed and interpreted the data and wrote the manuscript. All authors revised and approved the manuscript.

Funding information Open Access funding provided by Projekt DEAL. This study was funded by the Dietmar Hopp Foundation (Project Number 1DH1811306).

\section{Compliance with ethical standards}

Conflict of interest The authors declare that they have no conflict of interest.

Research involving human participants and/or animals The study followed the ethical standards of the Declaration of Helsinki, was approved by the Ethics Committee of the Medical Faculty of Heidelberg (S-347/2016), and is registered at clinicaltrials.gov (NCT02883699). This article does not contain any studies with animals performed by any of the authors.

Informed consent Informed consent was obtained from all individual participants included in the study.

Open Access This article is licensed under a Creative Commons Attribution 4.0 International License, which permits use, sharing, adaptation, distribution and reproduction in any medium or format, as long as you give appropriate credit to the original author(s) and the source, provide a link to the Creative Commons licence, and indicate if changes were made. The images or other third party material in this article are included in the article's Creative Commons licence, unless indicated otherwise in a credit line to the material. If material is not included in the article's Creative Commons licence and your intended use is not permitted by statutory regulation or exceeds the permitted use, you will need to obtain permission directly from the copyright holder. To view a copy of this licence, visit http://creativecommons.org/licenses/by/4.0/.

\section{References}

1. Schmitz KH, Courneya KS, Matthews C, Demark-Wahnefried W, Galvao DA, Pinto BM, Irwin ML, Wolin KY, Segal RJ, Lucia A, Schneider CM, von Gruenigen VE, Schwartz AL, American College of Sports M (2010) American College of Sports Medicine roundtable on exercise guidelines for cancer survivors. Med Sci Sports Exerc 42:1409-1426

2. Hayes SC, Spence RR, Galvao DA, Newton RU (2009) Australian Association for Exercise and Sport Science position stand: optimising cancer outcomes through exercise. J Sci Med Sport $12: 428-434$

3. Rock CL, Doyle C, Demark-Wahnefried W, Meyerhardt J, Courneya KS, Schwartz AL, Bandera EV, Hamilton KK, Grant B, McCullough M, Byers T, Gansler T (2012) Nutrition and physical activity guidelines for cancer survivors. CA Cancer J Clin 62: 243-274

4. Jones LW, Liang Y, Pituskin EN, Battaglini CL, Scott JM, Hornsby WE, Haykowsky M (2011) Effect of exercise training on peak 
oxygen consumption in patients with cancer: a meta-analysis. Oncologist 16:112-120

5. Ferguson B (2014) ACSM's guidelines for exercise testing and prescription, 9th edn. Canadian Chiropractic Association

6. Gil-Rey E, Quevedo-Jerez K, Maldonado-Martin S, HerreroRomán F (2014) Exercise intensity guidelines for cancer survivors: a comparison with reference values Int J Sports Med

7. Jones LW, Haykowsky M, Peddle CJ, Joy AA, Pituskin EN, Tkachuk LM, Courneya KS, Slamon DJ, Mackey JR (2006) Cardiovascular risk profile of breast cancer patients treated with anthracycline-taxane containing adjuvant chemotherapy and/or trastuzumab. J Clin Oncol 24:666-666

8. Scharhag-Rosenberger F, Kuehl R, Klassen O, Schommer K, Schmidt ME, Ulrich CM, Wiskemann J, Steindorf K (2015) Exercise training intensity prescription in breast cancer survivors: validity of current practice and specific recommendations. J Cancer Surviv 9:612-619

9. Courneya KS, McKenzie DC, Mackey JR, Gelmon K, Friedenreich CM, Yasui Y, Reid RD, Cook D, Jespersen D, Proulx C, Dolan LB, Forbes CC, Wooding E, Trinh L, Segal RJ (2013) Effects of exercise dose and type during breast cancer chemotherapy: multicenter randomized trial. J Natl Cancer Inst 105:1821-1832

10. Segal RJ, Reid RD, Courneya KS, Sigal RJ, Kenny GP, Prud'Homme DG, Malone SC, Wells GA, Scott CG, Slovinec D'Angelo ME (2009) Randomized controlled trial of resistance or aerobic exercise in men receiving radiation therapy for prostate cancer. J Clin Oncol 27:344-351

11. Beaver W, Wasserman K, Whipp B (1986) A new method for detecting anaerobic threshold by gas exchange. J Appl Physiol 60: 2020-2027

12. Coen B, Schwarz L, Urhausen A, Kindermann W (1991) Control of training in middle- and long-distance running by means of the individual anaerobic threshold. Int J Sports Med 12:519-524

13. Mann T, Lamberts RP, Lambert MI (2013) Methods of prescribing relative exercise intensity: physiological and practical considerations. Sports Med 43:613-625

14. Wolpern AE, Burgos DJ, Janot JM, Dalleck LC (2015) Is a threshold-based model a superior method to the relative percent concept for establishing individual exercise intensity? A randomized controlled trial BMC Sports Science. Med Rehab 7:16

15. Faude O, Kindermann W, Meyer T (2009) Lactate threshold concepts: how valid are they? Sports Med 39:469-490

16. Meyer T, Lucia A, Earnest CP, Kindermann W (2005) A conceptual framework for performance diagnosis and training prescription from submaximal gas exchange parameters - theory and application. Int J Sports Med 26:S38-S48

17. Cornette $\mathrm{T}$, Vincent $\mathrm{F}$, Mandigout $\mathrm{S}$, Antonini MT, Leobon S, Labrunie A, Venat L, Lavau-Denes S, Tubiana-Mathieu N (2016) Effects of home-based exercise training on $\mathrm{VO} 2$ in breast cancer patients under adjuvant or neoadjuvant chemotherapy (SAPA): a randomized controlled trial. Eur J Phys Rehabil Med 52:223-232

18. Travier N, Velthuis MJ, Steins Bisschop CN, van den Buijs B, Monninkhof EM, Backx F, Los M, Erdkamp F, Bloemendal HJ, Rodenhuis C, de Roos MA, Verhaar M, ten Bokkel HD, van der Wall E, Peeters PH, May AM (2015) Effects of an 18-week exercise programme started early during breast cancer treatment: a randomised controlled trial. BMC Med 13:121

19. Van Vulpen JK, Velthuis MJ, Steins Bisschop CN, Travier N, Van Den Buijs BJ, Backx FJ, Los M, Erdkamp FL, Bloemendal HJ, Koopman M, De Roos MA, Verhaar MJ, Ten Bokkel-Huinink D, Van Der Wall E, Peeters PH, May AM (2016) Effects of an exercise program in colon cancer patients undergoing chemotherapy. Med Sci Sports Exerc 48:767-775

20. Dickhuth HH, Yin L, Niess A, Röcker K, Mayer F, Heitkamp HC, Horstmann T (1999) Ventilatory, Lactate-derived and catecholamine thresholds during incremental treadmill running: relationship and reproducibility. Int J Sports Med 20:122-127

21. Jones LW, Courneya KS, Mackey JR, Muss HB, Pituskin EN, Scott JM, Hornsby WE, Coan AD, Herndon JE, Douglas PS, Haykowsky M (2012) Cardiopulmonary function and age-related decline across the breast cancer survivorship continuum. J Clin Oncol 30:2530 2537

22. Scharhag-Rosenberger F, Carlsohn A, Cassel M, Mayer F, Scharhag J (2011) How to test maximal oxygen uptake: a study on timing and testing procedure of a supramaximal verification test. Appl Physiol Nutr Metab 36:153-160

23. Borg GA (1982) Psychophysical bases of perceived exertion. Med Sci Sports Exerc 14:377-381

24. Coyle E, Martin W, Ehsani A, Hagberg J, Bloomfield S, Sinacore D, Holloszy J (1983) Blood lactate threshold in some well-trained ischemic heart disease patients. J Appl Physiol 54:18-23

25. Midgley AW, McNaughton LR, Carroll S (2006) Verification phase as a useful tool in the determination of the maximal oxygen uptake of distance runners. Appl Physiol Nutr Metab 31:541-548

26. Midgley AW, McNaughton LR, Polman R, Marchant D (2007) Criteria for determination of maximal oxygen uptake: a brief critique and recommendations for future research. Sports Med 37: $1019-1028$

27. Garber CE, Blissmer B, Deschenes MR, Franklin BA, Lamonte MJ, Lee IM, Nieman DC, Swain DP, American College of Sports M (2011) American College of Sports Medicine position stand. Quantity and quality of exercise for developing and maintaining cardiorespiratory, musculoskeletal, and neuromotor fitness in apparently healthy adults: guidance for prescribing exercise. Med Sci Sports Exerc 43:1334-1359

28. Van Schuylenbergh R, Vanden Eynde B, Hespel P (2004) Correlations between lactate and ventilatory thresholds and the maximal lactate steady state in elite cyclists. Int J Sports Med 25: 403-408

29. Rogers LQ, Markwell S, Hopkins-Price P, Vicari S, Courneya KS, Hoelzer K, Verhulst S (2011) Reduced barriers mediated physical activity maintenance among breast cancer survivors. J Sport Exerc Psychol 33:235-254

30. Scharhag-Rosenberger F, Meyer T, Gassler N, Faude O, Kindermann W (2010) Exercise at given percentages of $\mathrm{VO}_{2 \max }$ : heterogeneous metabolic responses between individuals. J Sci Med Sport 13:74-79

31. Gladden LB, Yates JW, Stremel RW, Stamford BA (1985) Gas exchange and lactate anaerobic thresholds: inter- and intraevaluator agreement. J Appl Physiol 58:2082-2089

32. Weatherwax RM, Harris NK, Kilding AE, Dalleck LC (2019) Incidence of $\mathrm{V} \mathrm{O}_{2 \max }$ responders to personalized versus standardized exercise prescription. Med Sci Sports Exerc 51:681-691

33. Poole DC, Jones AM (2017) Measurement of the maximum oxygen uptake $\dot{\mathrm{V}} \mathrm{O}_{2 \max }: \dot{\mathrm{VO}}_{2 \text { peak }}$ is no longer acceptable. J Appl Physiol 122:997-1002

34. Schaun GZ (2017) The maximal oxygen uptake verification phase: a light at the end of the tunnel? Sports Med Open 3:44

Publisher's note Springer Nature remains neutral with regard to jurisdictional claims in published maps and institutional affiliations. 